\title{
Silver Nanowire Diameter and Yield Characterization by High-Throughput SEM and Image Analysis
}

\author{
Clifford S Todd ${ }^{1}$, William A Heeschen ${ }^{1}$, Peter Y Eastman ${ }^{2}$, Ellen C Keene ${ }^{1}$. \\ 1. Analytical Sciences, The Dow Chemical Company, Midland MI, USA. \\ 2. Analytical Sciences, The Dow Chemical Company, Collegeville PA, USA.
}

An interconnected random network of nano-sized metal wires can be used as a Transparent Conductive Material [1]. TCMs are widely used in electronic devices from TVs and solar panels to touch screen applications such as tablets and phones. Potential advantages of such TCMs over incumbent indium tin oxide are lower sheet resistance at equal or better optical properties, low temperature deposition on polymer or other flexible substrates, cost advantages from capital expenditure reductions, and high throughput when applied by roll-to-roll coating. A scalable hydrothermal synthesis for silver nanowires $(\mathrm{AgNW})$ was developed [2,3] necessitating the efficient and statistically rigorous characterization of wire dimensions and wire-to-particle yield in order to track synthesis and purification developments.

Scanning electron microscopy was chosen as the imaging method for quantifying wire diameter distributions and wire/particle yield. Light microscopy does not have the spatial resolution to measure objects that are 10s of $\mathrm{nm}$; transmission electron microscopy has better spatial resolution than SEM, but is not amenable to a high-throughput workflow. Scanned probe microscopy was not used due to its smaller field of view, slower speed, and more interference from the polymer that may coat wires. Also AFM tip shape interactions tend to exaggerate wire diameters.

For each sample, a drop of aqueous AgNW suspension was placed on clean polished silicon wafer fragments and dried. The Si-wafers are flat and featureless, have a large atomic number contrast with silver, and are conductive enough that no metal sputter-coating is necessary for SEM imaging. A field emission gun SEM was used to collect backscattered electron images at $5 \mathrm{keV}$ with a pixel size of $3 \mathrm{~nm}$. This voltage is low enough to give good spatial resolution, yet large enough to give good signal to noise for the solid state BSE detector (Fig 1A). Automated stage movement and focus was used to acquire 18 images per sample on more than a dozen samples per night, unattended. Along with being an efficient way to collect SEM images, this reduced user bias by having only two of the 18 image locations chosen by the operator; there was no opportunity for the SEM operator to consciously or unconsciously choose fields of atypically thin or thick wires. The brightness and contrast settings were not changed during an automated image acquisition batch; the SEM working distance was also held constant. As a result, all images have a consistent gray-level for substrate and for silver, a key requirement for batch image analysis processing.

Image analysis was performed using in-house written macros for ImageJ software. After threshold as a mask, gray-level watershed segmentation created binary features that represent separated objects. Aspect ratio determined initial categorization for wire versus particle; a color-coded binary image was presented to the analyst to confirm or alter wire and particle determination (Fig 1B). Particle areas were tabulated (Fig 1D). Diameter calculation was done on isolated segments of wire. This involved creating a skeleton of the wire-only binary image, and using that to locate and remove wire crossings. The remaining skeleton was then used to measure the values along each wire segment from a Euclidean Distance Map of the wire-only binary image; this was converted to the average diameter of that wire 
segment. For batch processing, the macro used one image within the folder to set the gray-level threshold and applied this to the rest of the images in the folder. The length-weighted diameter of nanowires was calculated (Fig 1C) and used as a figure of merit for the resulting film properties. Wire/(Wire+Particle) by area was also calculated (Fig 1B).

During image analysis development, a panel of six people manually selected the threshold they thought most appropriate for each of eight test images from one sample. Depending on which judge and which image was selected to pick the threshold for the set, the resulting diameter average could vary up to about $15 \mathrm{~nm}$. Considering that the wires were only about $40 \mathrm{~nm}$ in diameter in this sample, threshold choice represents an enormous source of variability and operator bias in diameter measurement. This was addressed by using an algorithm for threshold instead of operator input. Of the 17 threshold algorithms available in ImageJ, all were applied to the eight images in the test set. The criteria used to evaluate the algorithms were: (1) Average threshold must be within the range picked by judges; (2) Variability in threshold between the images should be low; (3) Impact of focus-quality on diameter measurement should be low. Only one algorithm passed all the criteria, and was subsequently used for all analyses.

Analysis of reproducibility and repeatability was conducted regarding sample preparation, SEM operator, and image analysis operator. Results indicate differences in average diameter of just $4 \mathrm{~nm}$ could be routinely statistically resolved even though standard deviations were $\sim 15 \mathrm{~nm}$. Imaging and image analysis regularly handled over 12 samples per 24-hr while not impinging daytime manual use of the SEM for other projects.

\section{References:}

[1] S De et al, ACS Nano 3 (2009) p. 1767.

[2] J Lunn, A Malek. US Patent Application 20130283974 A1 (2013).

[3] GL Athens et al, TechConnect World conference (2015).

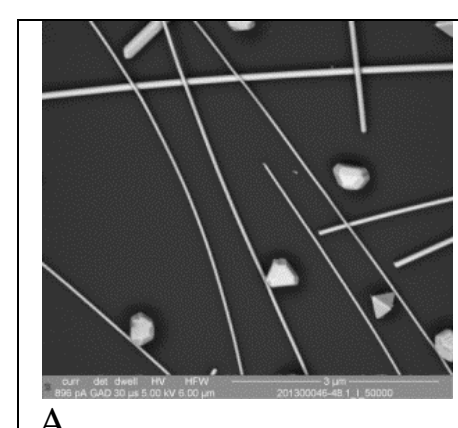

A
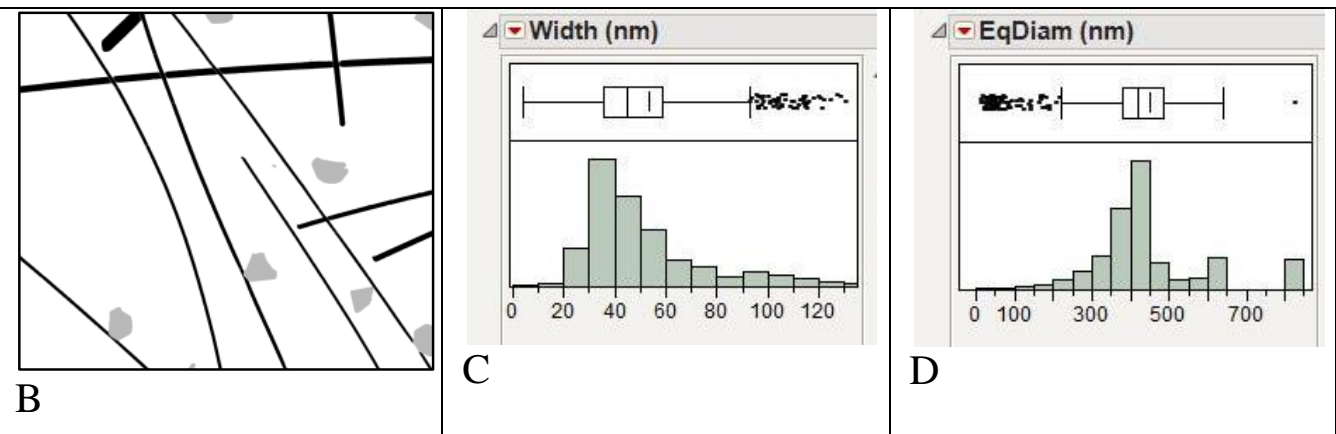

Figure 1. (A) One of 18 SEM image collected on a sample. Field of view is 6 microns. (B) Binary image part way through image analysis; wires in black, particles in gray. Wire yield is the black/(black+gray) area, 0.772 for the 18 images. (C) Wire diameter summary statistics for all 18 images of a sample. Mean diameter $=53.7 \mathrm{~nm}$, Median $=44.6 \mathrm{~nm}$, Standard Deviation $33.4 \mathrm{~nm}$. (D) Particle equivalent diameter summary statistics for the sample. Mean equivalent diameter $=448 \mathrm{~nm}$. 